\title{
Removal of trivalent chromium using hexane-extracted spent bleaching earth
}

\begin{abstract}
The removal of trivalent chromium from synthetic solution by hexane-extracted spent bleaching earth was investigated on a laboratory scale under various conditions. Data obtained followed the Langmuir isotherm for the $\mathrm{Cr}$ (III)-hexane extracted spent bleaching earth. Maximum sorption was calculated to be $9.48 \mathrm{mg} / \mathrm{g}$ at room temperature.
\end{abstract}

Keyword: Hexane-extracted spent bleaching earth; Sorption; Trivalent chromium 